\title{
Борновское приближение в задачах рассеяния на наночастицах
}

\author{
Д.А. Шапиро ${ }^{1,2}$, A.С. Берёза ${ }^{1, *}$ \\ ${ }^{1}$ Институт автоматики и электрометрии СО РАН \\ ${ }^{2}$ Новосибирский государственный университет \\ "E-mail: alex.bereza2010@yandex.ru
}

DOI:10.31868/RFL2018.95-96

Для современных технологий фотоники и наноплазмоники требуется расчет ближнего поля электромагнитной волны, рассеянной на частице. Только немногие задачи позволяют получить аналитическое решение в фотонике; таковым является классический случай рассеяния на одном цилиндре [1], рассеяние на двух цилиндрах [2] и двух идеально проводящих сферах [3] в квазистатическом пределе в биполярных координатах. Одним из способов получения аналитического решения является борновское приближение, суть которого заключается в том, чтобы для слабого рассеивателя вместо полного поля внутри рассеивающего потенциала использовать падающее поле. Однако, традиционный борновский ряд не позволяет точно учесть граничные условия в системам с резкой границей.

Целью данной работы является построение модифицированного борновского приближения для задачи рассеяния электромагнитной волны на нанообъектах. Для чего в задаче выделяют возмущенную и невозмущенную среды; Для последней должно быть известно решением в аналитическом виде, основываясь на котором, строится специальная функция Грина [4]. Тем самым задача сводится к возмутителю, помещенному в модифицированную среду, характеристики которой описываются специальной функцией Грина. После выводятся интегральные соотношения, связывающие внешнее поле и поле внутри возмутителя, из которых методом последовательных приближений получается решение в виде борновского ряда. Такое борновское приближение точно учитывает все граничные условия.

Данный метод был рассмотрен на примере задачи рассеяния электромагнитной волны на системе из двух параллельных цилиндров. Для случая s- и p- поляризаций была построена функция Грина и получена первая борновская поправка к рассеянному полю, а так же рекуррентные соотношения для нахождения последующих поправок. Оценен диапазон применимости решения. Проведено сравнение с двумя независимыми численными решениями: методом граничных элементов [4] и методом точечных диполей [5]. Первое борновское приближения подходит для определения качественного характера рассеяния, второе и последующие могут быть использованы для описания его количественных характеристик. Метод обобщен на случай большего числа тел произвольной формы. 


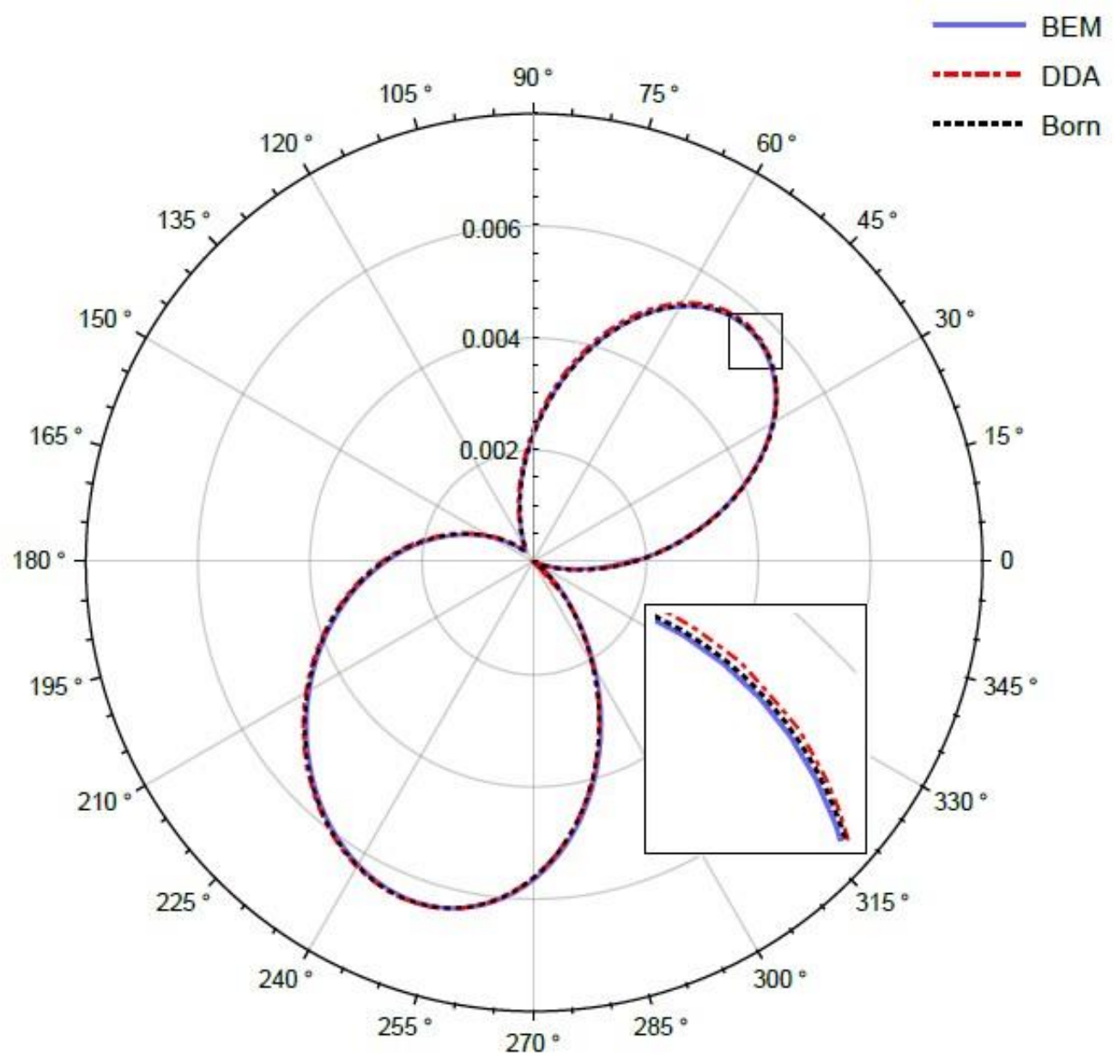

Рис.1. Угловая диаграмма для квадрата модуля рассеянного поля: ВЕМ - решение, полученное методом граничных элементов, DDA - методом точечных диполей, Born - решение с учетом третьего борновского приближения.

\section{Литература}

[1] L. Rayleigh, Philosophical Magazine, Series 6 36, 365-376 (1918).

[2] P. E. Vorobev, JETP 110, 193-198 (2010).

[3] I. E. Mazets, Technical Physics 45, 1238-1240 (2000).

[4] A.S. Bereza, A. V. Nemykin, S V. Perminov, L. L. Frumin and D. A. Shapiro, Phys. Rev. A, 95(6):063839, (2017).

[5] O. V. Belai, L. L. Frumin, S. V. Perminov, and D. A. Shapiro, EPL 97, (2012) 